\title{
The NGC 7771+NGC 7770 minor merger: harassing the little one?^
}

\author{
Almudena Alonso-Herrero, ${ }^{1, \dagger \ddagger ~ F . ~ F a b i a ́ n ~ R o s a l e s-O r t e g a, ~}{ }^{2,3}$ Sebastián F. Sánchez, ${ }^{3,4}$ \\ Robert C. Kennicutt, ${ }^{5}$ Miguel Pereira-Santaella ${ }^{6}$ and Ángeles I. Díaz ${ }^{2}$ \\ ${ }^{1}$ Instituto de Física de Cantabria, CSIC-UC, 39005 Santander, Spain \\ ${ }^{2}$ Departamento de Física Teórica, Universidad Autónoma de Madrid, 28049 Madrid, Spain \\ ${ }^{3}$ Centro Astronómico Hispano Alemán Calar Alto, CSIC-MPG, 04004 Almería, Spain \\ ${ }^{4}$ Instituto de Astrofísica de Andalucía, CSIC, 18080 Granada, Spain \\ ${ }^{5}$ Institute of Astronomy, University of Cambridge, Cambridge CB3 OHA \\ ${ }^{6}$ Istituto di Astrofisica e Planetologia Spaziali, INAF-IAPS, 00133 Rome, Italy
}

Accepted 2012 June 7. Received 2012 June 1; in original form 2012 April 20

\begin{abstract}
Numerical simulations of minor mergers, typically having mass ratios greater than 3:1, predict little enhancement in the global star formation activity. However, these models also predict that the satellite galaxy is more susceptible to the effects of the interaction than the primary. We use optical integral field spectroscopy and deep optical imaging to study the NGC 7771+NGC 7770 interacting system $(\sim 10: 1$ stellar mass ratio) to test these predictions. We find that the satellite galaxy NGC 7770 is currently experiencing a galaxy-wide starburst with most of the optical light being from young and post-starburst stellar populations $(<1 \mathrm{Gyr})$. This galaxy lies off the local star-forming sequence for composite galaxies with an enhanced integrated specific star formation rate. We also detect in the outskirts of NGC $7770 \mathrm{H} \alpha$ emitting gas filaments. This gas appears to have been stripped from one of the two galaxies and is being excited by shocks. All these results are consistent with a minor-merger-induced episode(s) of star formation in NGC 7770 after the first close passage. Such effects are not observed on the primary galaxy NGC 7771 .
\end{abstract}

Key words: galaxies: evolution - galaxies: individual: NGC 7770 - galaxies: individual: NGC 7771 - galaxies: nuclei - galaxies: structure - infrared: galaxies.

\section{INTRODUCTION}

Minor mergers are defined to have mass ratios greater than approximately $3: 1$. They have been increasingly recognized as important players in galaxy evolution and, in particular, in the formation and assembly of bulges especially in lower mass systems (see Hopkins et al. 2010, and references therein). Numerical simulations of minor mergers indicate that they can trigger nuclear activity and transform the morphologies of galaxies (Mihos \& Hernquist 1994; Hernquist \& Mihos 1995; Naab \& Burkert 2003; Robertson et al. 2006; Qu et al. 2011). The simulations of Cox et al. (2008) showed in detail that during the interaction process of minor mergers the global star

\footnotetext{
^ Based on observations collected at the Centro Astronómico HispanoAlemán (CAHA) at Calar Alto, operated jointly by the Max-Planck-Institut für Astronomie and the Instituto de Astrofísica de Andalucía (CSIC). The data presented here were obtained (in part) with ALFOSC, which is provided by the Instituto de Astrofísica de Andalucía (IAA) under a joint agreement with the University of Copenhagen and NOTSA.

†E-mail: aalonso@ifca.unican.es

$\ddagger$ Augusto González Linares Senior Research Fellow.
}

formation rate (SFR) only increases moderately and the increase is a function of the mass ratio of the galaxies. Furthermore, the fractional SFR enhancement is much higher in the satellite galaxy as it is more susceptible to the tidal forces induced by the interaction.

In this work we use optical imaging and integral field spectroscopy (IFS) to study the impact of the minor merger on the star formation properties of the individual galaxies of the NGC $7770+$ NGC 7771 system. We use these observations to test the predictions of minor-merger simulations. The primary galaxy NGC 7771 is an SB(s)a galaxy, the satellite galaxy NGC 7770 is classified as an S0/a (see Fig. 1) and they have an approximate stellar mass ratio of 10:1 (Pereira-Santaella et al. 2011). They are in a group with NGC 7769 to the north-west of the system and a small galaxy to the west of NGC 7771, with all of them being embedded in a common neutral hydrogen envelope (Nordgren et al. 1997). Throughout this work we assume a common distance of $60 \mathrm{Mpc}$.

\section{OBSERVATIONS}

We used the Potsdam Multi-Aperture Spectrograph (PMAS; Roth et al. 2005) in the PMAS fiber Package (PPAK) mode (Kelz et al. 2006) on the 3.5-m telescope in Calar Alto to observe the NGC 


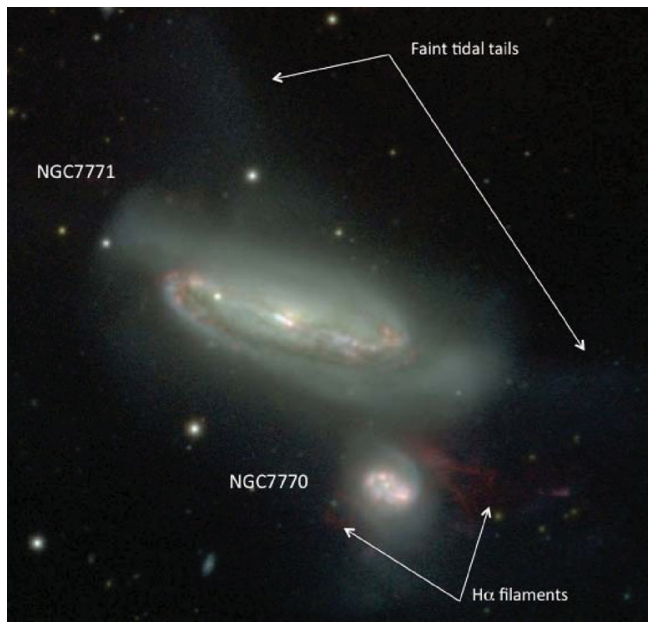

Figure 1. ALFOSC false-colour red, green and blue (RGB) image of the NGC $7771+$ NGC 7700 system constructed using the SDSS broad-band $g$ and $r$ images in blue and green, respectively, and the narrow-band $\mathrm{H} \alpha+[\mathrm{N}$ II] image in red. Orientation is north up and east to the left. The approximate FoV is $210 \times 200 \operatorname{arcsec}^{2}$. We also mark some morphological features discussed in Section 3.1.

$7771+$ NGC 7770 system. This was part of the PPAK IFS Nearby Galaxies Survey (PINGS). Very briefly, we used the V300 grating to cover the 3700-7100 $\AA$ spectral range with a spectral resolution of $10 \AA$. We took three pointings with a dithered pattern, which allowed us to resample the PPAK 2.7-arcsec-diameter fibre to a final mosaic with a 2-arcsec spaxel and a field of view (FoV) of $148 \times 130 \mathrm{arcsec}^{2}$. We reduced the data following Sánchez (2006). Before constructing the maps of the emission lines, we fitted the stellar continuum with the FIT3D routine (Sánchez et al. 2007). We used the Bruzual \& Charlot (2003) models to generate single stellar populations (SSPs) with a Salpeter initial mass function (IMF) covering a range of ages and metallicities. We refer the reader to Sánchez et al. (2011) for more details. We finally subtracted the fitted stellar continuum and used the observed
$\mathrm{H} \alpha / \mathrm{H} \beta$ line ratio to correct the line maps, on a spaxel-by-spaxel basis, for extinction (see Rosales-Ortega et al. 2010, for a full description).

We also obtained imaging with the Andalucia Faint Object Spectrograph and Camera (ALFOSC) on the 2.5-m Nordic Optical Telescope (NOT) in El Roque de los Muchachos. We used the Sloan Digital Sky Survey (SDSS) broad-band $g$ and $r$ filters and an $\mathrm{H} \alpha+\left[\mathrm{N}_{\mathrm{II}}\right]$ narrow-band filter $\left[\lambda_{\mathrm{c}}=6653 \AA\right.$ and $\Delta \lambda=55 \AA$ at full width half-maximum (FWHM)]. The instrument has a 0.19 arcsec pixel $^{-1}$ plate scale, and the observations were obtained under photometric and extremely good seeing $(\sim 0.6$ arcsec FWHM as measured from stars in the FoV) conditions. We followed standard techniques to reduce and calibrate the data, as well as to subtract the continuum from the $\mathrm{H} \alpha+\left[\mathrm{N}_{\mathrm{II}}\right]$ image and compute the $\mathrm{H} \alpha$ emission

\section{RESULTS}

\subsection{Optical continuum and ionized gas properties}

The deep ALFOSC optical continuum images allow us to get a detailed view of the morphology of the system, especially of the lesser known galaxy NGC 7770. Although NGC 7770 has been classified as an S0/a, it shows a faint spiral structure with bright knots located in a ring of star formation (Fig. 1). There are also other interesting features seen in the optical continuum emission such as a truncated stellar disc in NGC 7771 and faint short tidal tails in both galaxies. Such continuum tail features are predicted by simulations of minor mergers right after the first passage (Cox et al. 2008).

Both galaxies in the system show extended $\mathrm{H} \alpha$ emission over several kpc (Fig. 2). In NGC 7771 the $\mathrm{H} \alpha$ emission arises from the nuclear region of the galaxy, which is in fact a ring of star formation (see Alonso-Herrero et al. 2010, and references therein), from bright $\mathrm{H}$ II regions at the end of the bar and in the spiral arms and from the disc of the galaxy. NGC 7770 also shows a circumnuclear ring of star formation with an approximate diameter of $20 \operatorname{arcsec}(\sim 5.7 \mathrm{kpc})$. The rings of star formation in both galaxies show optical line ratios
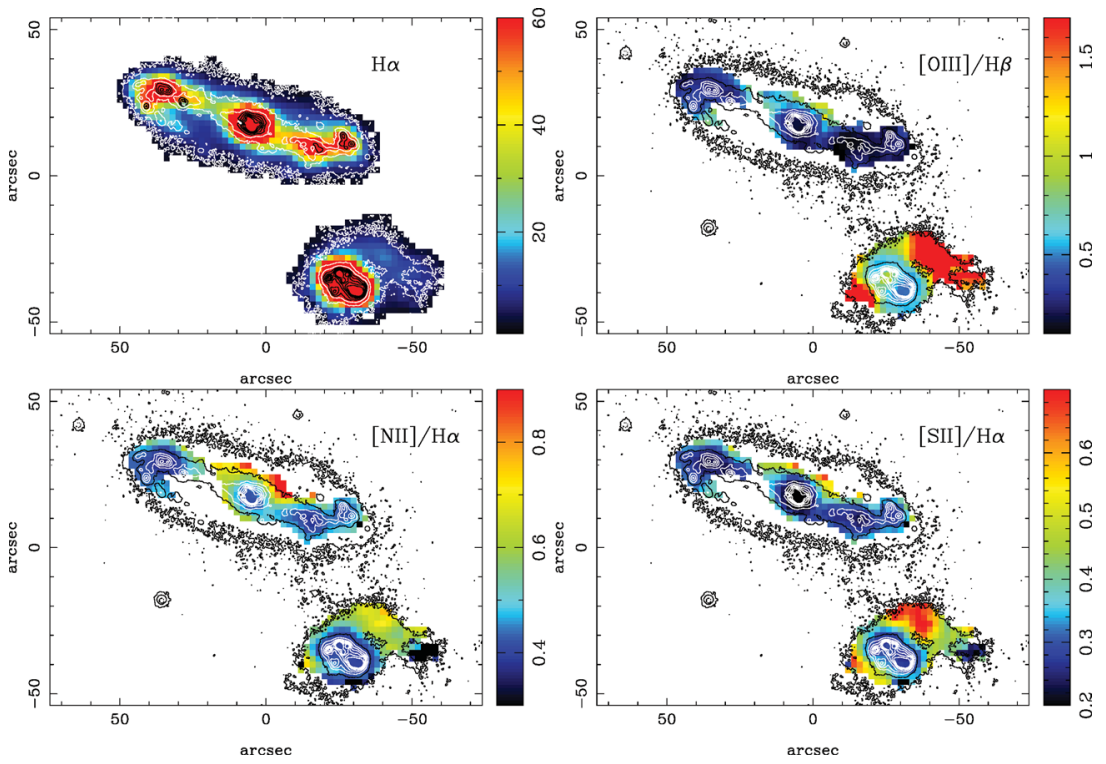

Figure 2. PPAK spectral maps (colour images) of the observed $\mathrm{H} \alpha$ emission (in units of $10^{-16} \mathrm{erg} \mathrm{cm}^{-2} \mathrm{~s}^{-1}$ arcsec ${ }^{-2}$ ) and the extinction-corrected $[\mathrm{O}$ III $] \lambda 5007 / \mathrm{H} \beta,\left[\mathrm{N}_{\mathrm{II}}\right] \lambda 6583 / \mathrm{H} \alpha$ and $\left[\mathrm{S}_{\mathrm{II}}\right] \lambda \lambda 6717,6731 / \mathrm{H} \alpha$ line ratios. The contours (in a square root scale) are the ALFOSC continuum-subtracted $\mathrm{H} \alpha$ emission. 

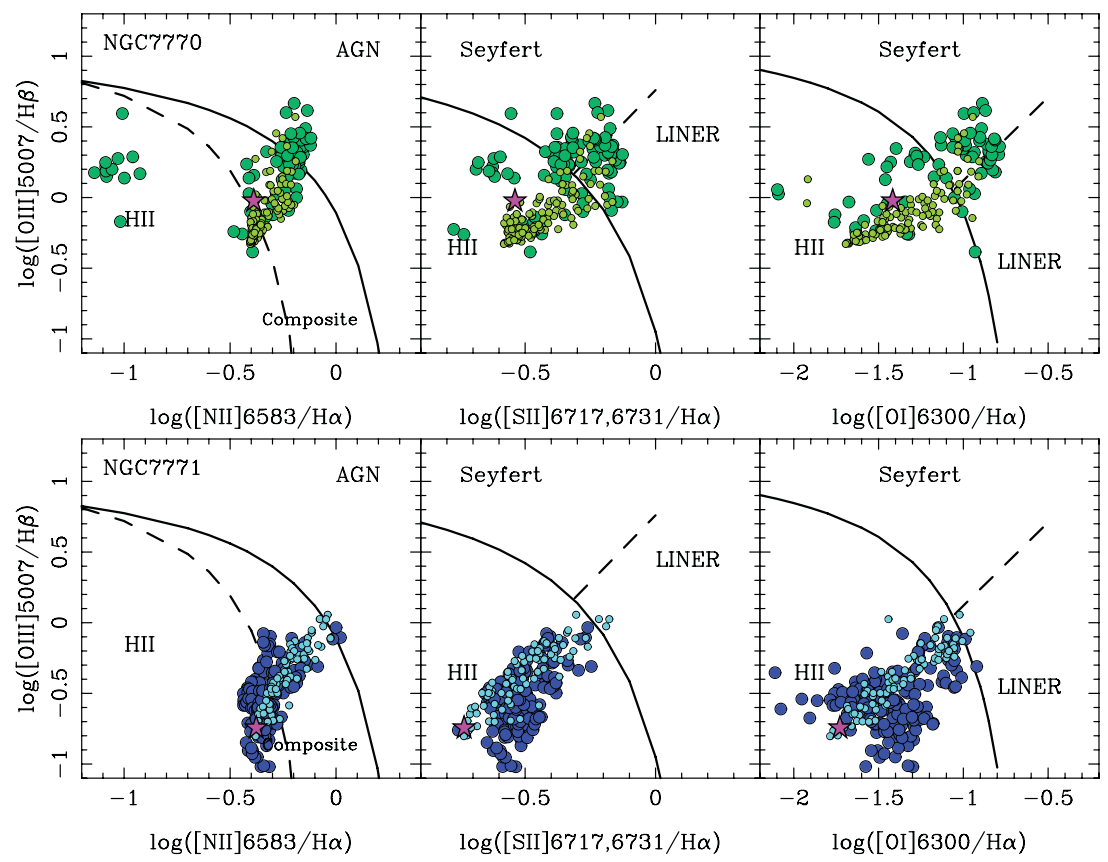

Figure 3. Spatially resolved diagnostic diagrams. Each measurement corresponds to one spaxel. The star-like symbols are the nuclear line ratios and the small and large dots are spaxels at projected galactocentric distances of $R<12 \operatorname{arcsec}$ and $R>12 \operatorname{arcsec}$, respectively. The solid curves are 'maximum starburst lines' (Kewley et al. 2001) and the dashed curves the empirical separation between composites and H II regions and Seyferts and LINERs (Kauffmann et al. 2003; Kewley et al. 2006). The spaxels of NGC 7770 (upper panel) in the LINER/Seyfert and AGN regions can also be produced by shocks (see text and also Allen et al. 2008).

typical of $\mathrm{H}$ II regions, as can be seen from the PPAK spectral maps (Fig. 2) and the spatially resolved diagnostic diagrams (Fig. 3). For the spaxels in both galaxies showing $\mathrm{H}$ II-region-like line ratios, the differences in the observed $[\mathrm{O}$ III $] / \mathrm{H} \beta$ line ratios are readily explained by differences in the gas-phase oxygen abundances (see Section 3.4).

The most striking feature is the filaments of diffuse $\mathrm{H} \alpha$ emission in the interface region between the two galaxies to the north-west of NGC 7770, and also to the south of the galaxy (see Fig. 1). These $\mathrm{H} \alpha$ filaments are not detected in the optical continuum emission (Fig. 1), indicating that they are mostly made of gas. The northwest filaments extend out to projected galactocentric distances of approximately $6 \mathrm{kpc}$. Minor-merger simulations (Cox et al. 2008) predict that gas is likely to have been cleared out from the primary galaxy extended disc after the first close passage of the satellite galaxy. The H $\alpha$ filaments of NGC 7770 and a few spaxels tracing the diffuse $\mathrm{H} \alpha$ emission around the ring of star formation of NGC 7771 (see also Alonso-Herrero et al. 2010) display elevated optical line ratios. This is better seen in the spatially resolved diagnostic diagrams (Fig. 3) where a large fraction of the outer (i.e. projected galactocentric distances $>12 \operatorname{arcsec} \sim 3.5 \mathrm{kpc}$ ) H $\alpha$ emitting regions of NGC 7770 are in the composite/active galactic nucleus (AGN) region or the Low Ionization Nuclear Emission line Region (LINER)/Seyfert region. The observed line ratios for the filament spaxels, even those falling in the LINER/Seyfert region, can be reproduced with the shock+precursor models of Allen et al. (2008) with shock velocities of $300-400 \mathrm{~km} \mathrm{~s}^{-1}$ (their figs 31-33). This could be understood if gas, from either NGC 7771 or NGC 7770, is being stripped and excited by shocks induced by tidal forces. In NGC 7771, there is no variation of the optical line ratios with the galactocentric distance.

\subsection{Luminosity-weighted stellar ages and metallicities}

The luminosity-weighted ages and metallicities of stellar populations can give us hints about the recent star formation history of galaxies. To ensure an appropriate modelling of the stellar populations, we used a Voronoi binning method (Cappellari \& Copin 2003) and the PINGSOFT (Rosales-Ortega 2011) software to define regions (also known as voxels) in the PPAK data cube with extracted spectra of sufficient signal-to-noise ratio $(\mathrm{S} / \mathrm{N})$ values. We targeted $\mathrm{S} / \mathrm{N}$ values of 25 over a spectral region centred at $5200 \AA$ with a $100 \AA$ width. We then used the FIT3D routine to fit SSPs to the entire spectrum of each voxel with a $\chi^{2}$ minimization technique. We generated a grid of templates using the Bruzual \& Charlot (2003) models with a range of ages $(5,25,100$ and $640 \mathrm{Myr}$, and 1.4, 2.5, $5,13$ and $17 \mathrm{Gyr})$ and three metallicities $(Z=0.008,0.02$ and 0.05$)$, and allowed for the templates to be attenuated. In general, it was not possible to distinguish among the few best-fitting models. We therefore estimated the age and metallicity of each extracted spectrum by averaging the ages and metallicities of these models, weighted by their corresponding $\chi^{2}$ values. For the following discussion, we grouped the stellar populations into young and post-starburst with ages of $<1 \mathrm{Gyr}$, intermediate with ages of $1-5 \mathrm{Gyr}$ and old with ages $>5$ Gyr.

The optical light of the primary galaxy is mostly dominated by intermediate and old stellar populations (see Fig. 4) with supersolar metallicities $(Z>0.025)$. There is a small contribution $(\sim 20$ per cent) from stellar populations with ages of $<1 \mathrm{Gyr}$, which are mostly located in the circumnuclear ring of star formation. This agrees with the presence of post-starburst stellar populations in the nuclear region of NGC 7771 found by previous works (see Davies, Alonso-Herrero \& Ward 1997; Alonso-Herrero et al. 2010). In the 

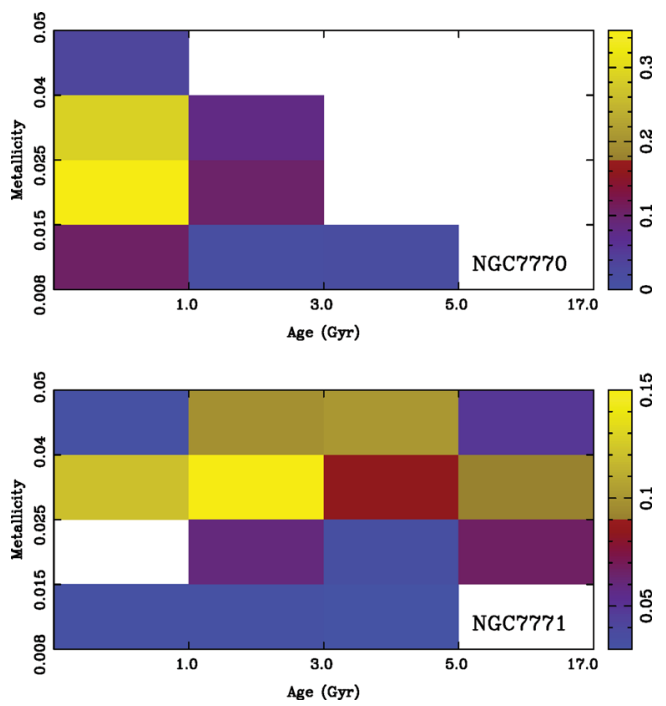

Figure 4. Distributions of the ages and metallicities of the spatially resolved (i.e. corresponding to the extracted spectra for the defined voxels, see text) modelled SSPs. The colours represent the fractional contribution of the SSP bins (luminosity weighted) to the observed optical emission (3700-7100 $\AA$ ) of each galaxy.

satellite galaxy NGC 7770, the young stellar populations ( $<1 \mathrm{Gyr}$ ) with solar and supersolar metallicities $(Z=0.015-0.04)$ contribute as much as $\sim 80$ per cent of the optical light.

The presence of a post-starburst stellar population has often been interpreted as the result of a past interaction (e.g. Liu \& Kennicutt 1995). The fact that the optical emission of NGC 7770 is dominated by this population suggests that the galaxies have already experienced first passage. This likely triggered a strong burst of star formation in the satellite galaxy less than $1 \mathrm{Gyr}$ ago, as predicted by the simulations of Cox et al. (2008). The origin of the post-starburst population in NGC 7771 is less clear since these minor-merger models do not predict a strong star formation enhancement in the primary. Based on the neutral hydrogen morphology, Nordgren et al. (1997) suggested that NGC 7771 and NGC 7769, which have a 2:1 mass ratio, appear to be having a prograde-retrograde interaction, with NGC 7769 being the retrograde one. Such an interaction, while not as efficient at triggering strong episodes of star formation, may explain the circumnuclear burst of star formation in NGC 7771.

\subsection{Current star formation activity}

Next we explore the impact of the interaction on the current SFR of the galaxies. For a 10:1 stellar mass ratio, Cox et al. (2008) predict only a mild total enhancement ( $<2$ times) in the global SFR of the system when compared to the sum of SFRs of the individual galaxies if they were isolated. In fact, most of the SFR enhancement takes place in the satellite galaxy because it is experiencing significant tidal forces. We can test this prediction for the individual galaxies of this system since in the local Universe star-forming galaxies define a sequence of increasing SFR for increasing stellar masses (e.g. Salim et al. 2007, and references therein).

The integrated extinctions $A_{V}$ of the galaxies (1.1 and $2.5 \mathrm{mag}$ for NGC 7770 and NGC 7771, respectively, from the $\mathrm{H} \alpha / \mathrm{H} \beta$ ratio) indicate that we can use the SFR recipe of Kennicutt et al. (2009). It combines the observed (not corrected for $A_{V}$ ) $\mathrm{H} \alpha$ luminosity and the infrared (IR) luminosity to account for the unobscured and the obscured SFRs, respectively. The observed $\mathrm{H} \alpha$ luminosities are
$L(\mathrm{H} \alpha)=2.7 \times 10^{41}$ and $4.2 \times 10^{41} \mathrm{erg} \mathrm{s}^{-1}$ for NGC 7770 and NGC 7771 , respectively, and the IR luminosities are $\log \left(L_{\mathrm{IR}} / \mathrm{L}_{\odot}\right)=10.8$ and 11.3 (Pereira-Santaella et al. 2011).

There is some evidence of the presence of an AGN in NGC 7770 both from the composite nuclear (i.e. star-forming/AGN) $[\mathrm{N} \mathrm{II]/H} \alpha$ line ratio (Fig. 3) and from the hot dust emission detected in the Spitzer spectrum (Alonso-Herrero et al. 2012). To compute the SFR we then need to subtract the AGN contribution from the IR luminosity. For a Kroupa IMF, we obtained SFR(total) $=4.5$ and $12.8 \mathrm{M}_{\odot} \mathrm{yr}^{-1}$ for NGC 7770 and NGC 7771, respectively. These are similar, within the uncertainties of the SFR recipes $( \pm 0.3 \mathrm{dex}$ or better), to those obtained from the extinction-corrected $L(\mathrm{H} \alpha)$. We note that the SFR recipe used here assumes that approximately half of the dust heating arises from stars older than $30 \mathrm{Myr}$. The IRbased calibration of Kennicutt (1998), which assumes that the dust is only heated by these young stars, results in SFRs approximately two times higher.

The specific SFRs $\left(\mathrm{SSFR}=\mathrm{SFR} / M_{*}\right)$ using stellar masses computed with a near-IR mass-to-light ratio (Bell \& de Jong 2001; Pereira-Santaella et al. 2011) are $\log \left(\mathrm{SSFR} / \mathrm{yr}^{-1}\right)=-9.6$ and -10.4 for NGC 7770 and NGC 7771, respectively. If there was no AGN in NGC 7770 then the calculated SFR and SSFR would be lower limits. We find that NGC 7771 falls on the high-mass end of the local star-forming sequence. Composite galaxies and AGN follow a similar star-forming sequence but extending to higher stellar masses (fig. 18 in Salim et al. 2007). The satellite galaxy NGC 7770 shows an elevated SSFR and lies just off the distinct region occupied by local composite galaxies. This suggests that not only does NGC 7770 show evidence of a post-starburst stellar population, but also it is currently undergoing a galaxy-wide burst of star formation. These results agree with predictions of minor-merger simulations as well as with statistical observational studies of minor mergers (Woods \& Geller 2007; Ellison et al. 2008).

\subsection{Gas-phase oxygen abundances}

Interacting galaxies are believed to contribute to the observed scatter in the local mass-metallicity relation (Tremonti et al. 2004). Specifically, local luminous and ultraluminous IR galaxies, which include a large fraction of gas-rich mergers, show lower nuclear abundances than local emission-line galaxies of similar luminosity and mass (Rupke, Veilleux \& Baker 2008). Simulations of interacting galaxies show that it is possible to decrease the nuclear metallicity although the gas content of the galaxies plays a role (Torrey et al. 2012).

We used the calibration of Pettini \& Pagel (2004) based on the O3N2 index to derive the oxygen abundances of NGC 7770 and NGC 7771 on a spaxel-by-spaxel basis, but only for regions with $\left[\mathrm{O}_{\mathrm{I}}\right] / \mathrm{H} \alpha<0.06$. In doing so, we ensured that most of the line emission used to compute the abundances is produced by photoionization in $\mathrm{H}$ II regions rather than by other mechanisms. The oxygen abundances of the regions of NGC 7770 are lower that those of NGC 7771 (Fig. 5) as expected from the different stellar masses and in agreement with the SSP modelling. The distribution of the oxygen abundance in NGC 7771 is rather complex with the $\mathrm{H}$ II regions at the east end of the bar having apparently lower abundances than those to the west. The abundance in the central regions of NGC 7770 appears to be about 0.1 dex lower than in the bright $\mathrm{H}$ II region to the south-west of the nucleus and in the outskirts of the galaxy. This may occur if gas from the outskirts of the satellite galaxy has been channelled to the nuclear region as the result of the interaction. 


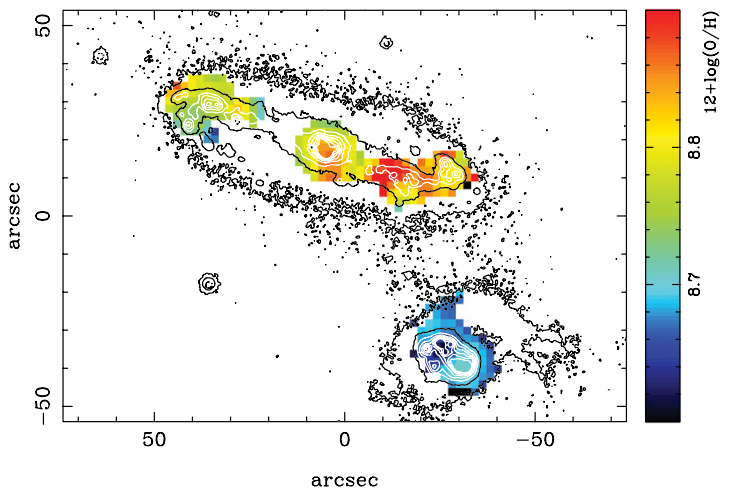

Figure 5. Map of oxygen abundances derived with the O3N2 index and the calibration of Pettini \& Pagel (2004) only for spaxels with no evidence of shock excitation ([O I $] \lambda 6300 / \mathrm{H} \alpha<0.06)$. Contours are as in Fig. 2.

However, the suspected composite nature of the nucleus of NGC 7770 may complicate this interpretation.

\section{CONCLUSIONS}

We presented PPAK spatially resolved optical emission-line ratio and abundance maps and diagnostic diagrams as well as ALFOSC deep optical imaging of the NGC 7771+NGC 7770 system to study the impact of the interaction on the star formation properties of the individual galaxies. The galaxies have a $\sim 10: 1$ stellar mass ratio and thus are classified as a minor merger. Numerical simulations of minor mergers predict little enhancement of the global star formation properties. The enhancement mostly affects the satellite galaxy. We find that the satellite galaxy in the system NGC 7770 is indeed experiencing a galaxy-wide starburst with most of its optical emission being produced by young and post-starburst stellar populations $(<1$ Gyr). This galaxy shows an enhanced SSFR with respect to the distinct region occupied by composite objects in the local star-forming sequence. We also detected $\mathrm{H} \alpha$ emitting gas filaments in the outskirts of NGC 7770 at projected galactocentric distances greater than $\sim 4 \mathrm{kpc}$ and out to $6 \mathrm{kpc}$. These filaments appear to be made of gas that has been stripped from one of the two galaxies and is being excited by shocks. Overall, our findings support the picture that the minor merger induced one or several episodes of star formation in the satellite galaxy NGC 7770 after the first close passage. Such interaction-induced effects are not observed in the primary galaxy of the system.

\section{ACKNOWLEDGMENTS}

We thank the referee for comments that helped improve the Letter and M. Cappellari for interesting discussions. The authors acknowledge financial support from the Spanish Plan Nacional grant AYA2010-21161-C02-01 and the Universidad de Cantabria AGL programme (AA-H), from grant AYA2010-21887-C04-03 (AID) and from the Mexican National Council for Science and Technology (CONACYT) through the programme Estancias Posdoctorales y Sabáticas al Extranjero para la Consolidación de Grupos de Investigación, 2010-2011 (FFR-O). MP-S is funded by an ASI fellowship under contract I/005/11/0.

\section{REFERENCES}

Allen M. G., Groves B. A., Dopita M. A., Sutherland R. S., Kewley L. J., 2008, ApJS, 178, 20

Alonso-Herrero A., García-Marín M., Rodríguez Zaurín J., Monreal-Ibero A., Colina L., Arribas S., 2010, A\&A, 522, A7

Alonso-Herrero A., Pereira-Santaella M., Rieke G. H., Rigopoulou D., 2012, ApJ, 744, 2

Bell E. F., de Jong R. S., 2001, ApJ, 550, 212

Bruzual A. G., Charlot S., 2003, MNRAS, 344, 1000

Cappellari M., Copin Y., 2003, MNRAS, 342, 345

Cox T. J., Jonsson P., Somerville R. S., Primack J. R., Dekel A., 2008 , MNRAS, 384, 386

Davies R. I., Alonso-Herrero A., Ward M. J., 1997, MNRAS, 291, 557

Ellison S. L., Patton D. R., Simard L., McConnachie A. W., 2008, AJ, 135, 1877

Hernquist L., Mihos J. C., 1995, ApJ, 448, 41

Hopkins P. F. et al., 2010, ApJ, 715, 202

Kauffmann G. et al., 2003, MNRAS, 346, 1055

Kelz A. et al., 2006, PASP, 118, 129

Kennicutt R. C., Jr, 1998, ARA\&A, 36, 189

Kennicutt R. C., Jr, et al., 2009, ApJ, 703, 1672

Kewley L. J., Dopita M. A., Sutherland R. S., Heisler C. A., Trevena J., 2001, ApJ, 556, 121

Kewley L. J., Groves B., Kauffmann G., Heckman T., 2006, MNRAS, 372, 961

Liu C. T., Kennicutt R. C., Jr, 1995, ApJ, 450, 547

Mihos J. C., Hernquist L., 1994, ApJ, 425, L13

Naab T., Burkert A., 2003, ApJ, 597, 893

Nordgren T. E., Chengalur J. N., Salpeter E. E., Terzian Y., 1997, AJ, 114, 77

Pereira-Santaella M. et al., 2011, A\&A, 535, A93

Pettini M., Pagel B. E. J., 2004, MNRAS, 348, L59

Qu Y., Di Matteo P., Lehnert M. D., van Driel W., Jog C. J., 2011, A\&A, 535, A5

Robertson B., Bullock J. S., Cox T. J., Di Matteo T., Hernquist L., Springel V., Yoshida N., 2006, ApJ, 645, 986

Rosales-Ortega F. F., 2011, New Astron., 16, 220

Rosales-Ortega F. F., Kennicutt R. C., Sánchez S. F., Díaz A. I., Pasquali A., Johnson B. D., Hao C. N., 2010, MNRAS, 405, 735

Roth M. M. et al., 2005, PASP, 117, 620

Rupke D. S. N., Veilleux S., Baker A. J., 2008, ApJ, 674, 172

Salim S. et al., 2007, ApJS, 173, 267

Sánchez S. F., 2006, AN, 327, 850

Sánchez S. F., Cardiel N., Verheijen M. A. W., Martín-Gordón D., Vilchez J. M., Alves J., 2007, MNRAS, 376, 125

Sánchez S. F. et al., 2011, MNRAS, 410, 313

Torrey P., Cox T. J., Kewley L., Hernquist L., 2012, ApJ, 746, 108

Tremonti C. et al., 2004, ApJ, 613, 898

Woods D. F., Geller M. J., 2007, ApJ, 134, 527

This paper has been typeset from a $\mathrm{T}_{\mathrm{E}} \mathrm{X} / \mathrm{LAT}_{\mathrm{E}} \mathrm{X}$ file prepared by the author. 